\title{
Exploration of Product Design Based on Behavioral Interaction and Emotional Guidance
}

\author{
Zhou Jun, Guo Shaokang \\ School of Design Art, Nanhua University, Hengyang, Hunan, China, 421001
}

Keywords: Behavioral Interaction; Emotional Guidance; Interactive Product Design; Experience

\begin{abstract}
This paper is to explore the value and design principles of behavioral interaction and emotional guidance in product design. Based on behavioral interaction and emotional design theory, cognitive behavioral interaction and emotional guidance are derived from the apocalyptic perspectives of psychology, physiology and emotional design, and their value in product design is analyzed combined with design cases. The PAMIP design analysis method centered on user research is proposed, and the deep connection between interaction behavior and emotion is explored through design practice. The three feasibility principles in product design practice are summarized as follows. (1) Strengthen the interaction mode of existing behavior and improve interaction efficiency (2) Use multi-directional interaction logic and be close to user perception to achieve experience goals. (3) Establish a good dialogue relationship between users and products and achieve a virtuous circle of interactive experience.
\end{abstract}

\section{Introduction}

Today, users have more expectations for the non-material elements, emotional experience, and value of the product. Therefore, the design needs to conduct an in-depth analysis of the behavioral interaction between people and products. Relying on the guidance of emotional elements, it effectively triggers behavioral interaction and information exchange between people and products in a more intimate way. While improving the product experience, it promotes the benign behavioral interaction and emotional resonance of people and products. The author tries to explore the feasible path of constructing good behavioral interaction and emotional experience in products based on behaviors, emotions, interactions, experiences and other perspectives.

\section{Interpret Behavioral Interaction}

Behavioral interaction is a kind of "people-oriented" natural interaction, aiming at interaction and communication through the presentation of behavioral actions and related feedback. Construct the interaction between people and products by observing and studying the most natural behavioral habits in the user's life. Behavioral interaction is seen as a way of guiding product interaction design, focusing on the behavioral interaction between people and products in product interaction design [1]. And this effect is used as a prototype theory to continuously expand into the practice of product 
interaction design.

\subsection{Design Theory of Behavioral Interaction}

The study of human behavior as a research object of interaction design has been around for a long time. Alan Cooper believes that a good interaction design should fully study user behavior and respect user goals, and follow the "user behavior and goal-oriented" design approach to achieve the actual needs of users as the ultimate goal. Professor Dan Boyaski divided the development process of interaction design into three time nodes: 1984, 1995 and 2013, which corresponded to three research objects: interface, behavior and experience. Bill Moggeridge and Bill Verplank proposed in 1985 that the research object of interactive interface design is not only limited to the content on the screen, but also includes people's usage behavior and corresponding use situation. Alan Cooper also believes that interaction design has to deal with some visual form problems, but the main design object is still behavior. Domestic research on behavioral interactions is relatively late. Professor Xin Xiangyang believes that the focus of interaction design and research objects should shift from people and machines to human behavior. Interaction design changes from physical logic to behavioral logic, from the rational allocation of the essential attributes of objects to the rational organization of behavioral attributes [2]. And define people, actions, tools or media, goals, and scenarios as the five elements of behavioral interaction. Professor Zhao Jianghong puts forward the "automatic behavior" psychological switch to understand the relationship between people and materials on the subconscious and behavioral habits of interaction design. He believes that behavioral interaction design should be more natural and close to users.

In summary, the deep understanding of behavior is the key to handling the product interaction relationship and the prerequisite for building a good product experience. The behavioral interaction must take into account the commonality and invariant of the behavior itself, and must take into account the differences and variables of different users in different situations. Under the new normal of technological development, the research on the subdivision of behavioral interaction has become more urgent. As a kind of interaction based on human behavior, understanding behavior from the psychological and physiological aspects of causing behavior helps us to better understand the intrinsic relationship between behavior and emotional interaction.

\subsection{Understand Behavioral Interaction}

John Broadus Watson, founder of behavioral psychology, believes that the object of psychological research is not consciousness but behavior [3]. That is, it is a process that through external behavior to speculate internal psychology. Psychology is sometimes even called the science of research behavior, which is the basis of cognitive behavior and interaction. Behavioral interaction is a system interaction method consisting of two-way behavior, which presupposes behavior and covers all the characteristics of behavior. The functioning of the human behavior system is conscious and exhibits different levels of consciousness [4]. It includes consciousness, subconsciousness and pre-consciousness, while different levels of consciousness correspond to different ways of behavior interaction. For example, the conscious behavioral interaction means that the user needs to use the training, reflection and other methods to interact with the product when using the product. The subconscious behavioral interaction is a behavioral interaction method based on user instinctive conditional reflection. Pre-conscious behavioral interactions are in between, and the way they interact depends on their own memories and experiences.

Behavioral physiologist Carlson believes that almost all human behavior is determined by the external environmental stimuli it accepts and its own motives. Motivation is an internal factor in which a person produces a specific behavior, which depends on the combined effects of the nervous 
and endocrine systems [5]. Humans maintain the steady state of their internal system environment by adjusting behavior patterns and the environment. The changing living environment requires people to constantly perceive the outside world and constantly adjust their behavior to adapt to the external environment. The nervous system, as the basis for generating behavioral activities, receives nerve impulses (inputs) that act on the external environment and act on the muscles, exhibiting rapid behavioral responses (outputs). The difference between the endocrine system and the nervous system is that it relies on endocrine cells to produce hormones that act on specific target cells to affect people's emotions, perceptions, motivations, etc., and ultimately achieve effects on human behavior and even control [6].

The facts show that human behavior is intertwined with many psychological and physiological factors. Psychology and physiology are both interrelated and independent of behavior, thus creating the complexity of behavior. Nowadays, when the product information features are significant and the interaction behavior is increasingly complex, how to construct a clear, concise and usable behavioral interaction method from complex behaviors to guide the product interaction design practice becomes more and more important.

\subsection{Build Behavioral Interaction}

From human and product perspective, Richard Buchanan believes that human behavior can be created and supported through the media role of a product (physical, virtual, service or system). A product is both an object of behavioral interaction and a material carrier for behavioral interaction. On the one hand, behavioral interaction, as a kind of "people-oriented" interaction, needs to pay attention to, study and understand human behavior and needs. Relevance and matching of people's subconscious behaviors with product use "behavior" enhances the fluency and resonance of people in the dynamic process of using products. On the other hand, the purpose of behavioral interaction is to create a good experience. Jesse James Garrett defines the user experience that how products interact with the outside world and work, that is, how people approach and use the product[7]. We can simplify the interactive process and improve the interactive experience by observing the actual interaction between people and products and combing the key behaviors in the interaction. Secondly, while paying attention to user behavior, product behavior should also be paid attention to. Products can rely on different interactive media such as sound and image to form different feedback effects. Figure 1 is SPIRO-PLUS lung function test device. This design gamifies the testing process by providing a familiar task (blowing a candle). The user's attention is transferred from the lung function test to the gamification situation. The simple and clear operation mode and LED light feedback, and friendly and smooth behavior interaction mode brings the user's intimate situational experience while improving the detection efficiency.

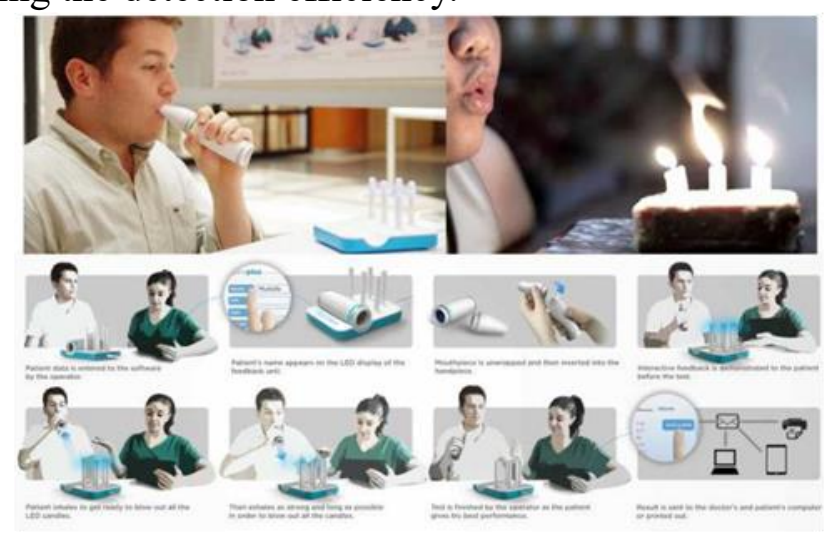

Figure 1 SPIRO-PLUS 
From the perspective of people, products and environment, people will have different behavioral responses in different scenarios, and good scene settings can lead to positive behavioral interactions. The coordination of products and the environment is also the basis for building good scenarios. For different users, the study of different scene modes is another key point to refine the interaction behavior and optimize the interaction quality to create a good experience.

\section{Emotional Guidance in Behavioral Interaction}

Emotional design is based on the premise of ensuring basic functions, with the primary purpose of understanding people's inner feelings and spiritual needs, and designing the behavioral activity process, so that users can further enhance the emotional experience when using products. It is relatively passive in the transmission of emotions. Emotional guidance is based on emotional design, and uses the revelation of emotional design to guide the subject to convey emotions to the object in an active way. From emotional design to guidance, it is a process of becoming passive and active. Emotional guidance creates an immersive emotional interaction experience by embedding emotional elements in the design to stimulate the interaction of emotion and behavior between people and products.

Emotional guidance is explored as a guided behavior. It is not only a kind of deepening of the passive emotional interaction, but also through the design of behavioral actions to guide people to convey emotions in an active attitude and enhance the emotional communication between people and people. Thereby, it gives the product a higher emotional added value, and strengthen the sense of context substitution in the process of interaction between people and products. As shown in Figure 2, "Touchables" is a couple interactive creative clothing design. Through the study of the couple's daily intimate behavior, the design is set up in pockets where the couples hug and shoulder-to-shoulder contact frequently. A subtle detail treatment is not only the strengthening and guiding of the couple's potential behavior, but also the interactive invitation signal to encourage the couple to have physical contact with each other. The design invisibly enhances the intimacy between the couples. "Touchables" guided design and positive behavioral interaction brings users a delicate and warm experience.

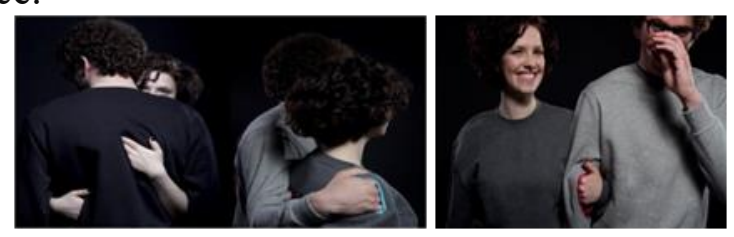

Figure 2 Touchables

\section{The Framework and Practice of Emotional Guidance in Behavioral Interaction}

The emotionally guided interactive design theory framework is based on user research, with products as the carrier, emotional support, and guided interaction to build products with good experience value. PAMIP design analysis method is based on behavioral interaction, emotional guidance theory and design practice. As shown in Figure 3, first, P1 (Person): Through user analysis, the user group is determined to achieve accurate positioning of the user. Secondly, A (Action): Analyze the user behavior and motivation, and design the behavior interaction mode. M (Mind): Analyze the emotional drivers implied by the user's daily behavior patterns for the design of emotional guidance. Furthermore, I (Interaction): Match behavioral interactions and emotional orientation factors, analyze and determine the feasibility of interaction, and design the interaction mode. Finally, P2 (Product): Analyze the current status and design feasibility of the product, position the product design. P1 (Person): Combine user testing, collect user feedback, and use it for 
iteration and innovation of emotionally guided products.
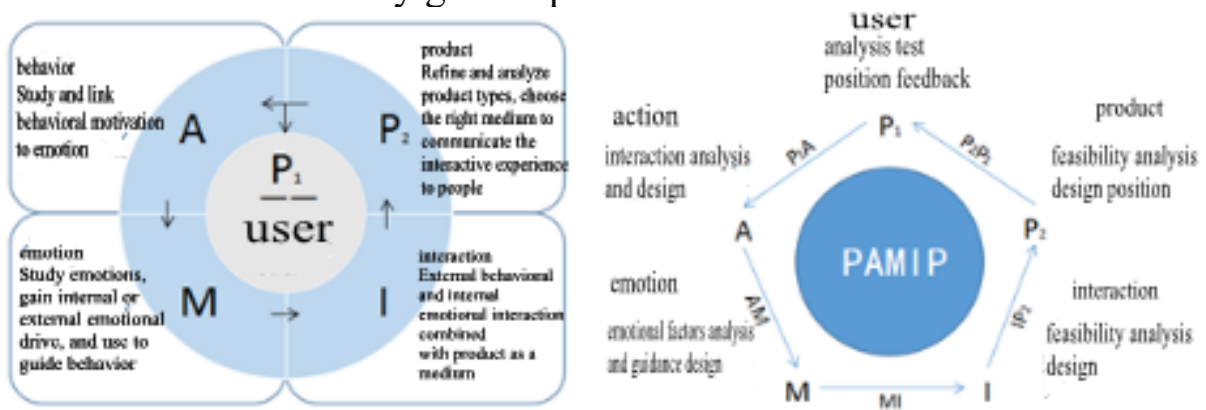

Figure 3 PAMIP design analysis framework

The author analyzes the design practice through "smile" and "touch". Figure 4 is the mirror of the author's gold award "Smirror" opened with "smile". Through observational studies of people with social depression, it has been found that long-term depression can cause troubles and stress, which is harmful to the health of people. The "Smirror" design reinforces the daily behavior of people smiling to the mirror, encouraging positive smiles with positive psychological cues, and positive emotion output in the interaction of people and mirrors. The product uses the face shape to bring people closer to the product. The mirror built-in face recognition camera can sense the user's facial expression. When the user opens the mirror with "smile", the light signal lights up and the mirror changes from blur to clear. At the same time, the user interacts with the "Smirror" through the smile behavior, and the mirror visually presents the positive emotion brought by the smile to the user. It will stimulate the emotional resonance and experience of the user, potentially guide the user's positive and optimistic life emotions, which is conducive to creating a social atmosphere of mutual friendliness and trust.

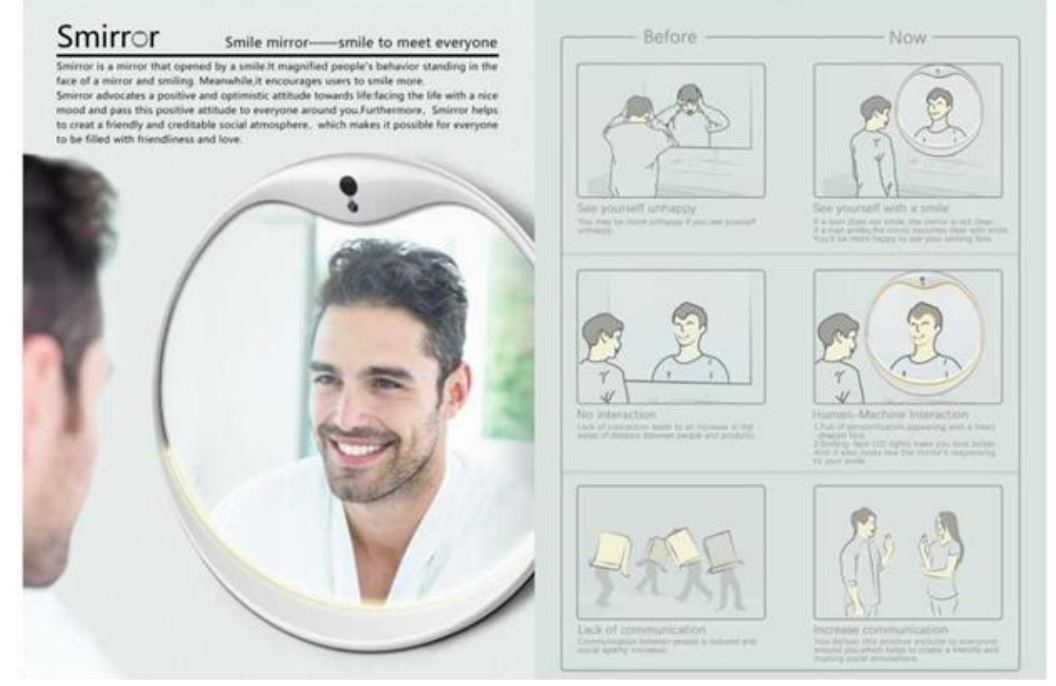

Figure 4 “Smirror” Smile Mirror 2017 China Pioneer Prize Gold Award Works (SIX Studio)

Practice shows that through PAMIP design analysis, the theme of "Emotion Guide Behavior and Behavior Enhance Emotion" is the core theoretical framework of emotional guidance and behavioral interactive product design. Emotional guided interactive product design is an effective path to create a benign multi-directional interaction and high emotional experience, and is the basis for practicing emotional interactive design practice. 


\section{Design Principles of Behavioral Interaction and Emotional Guidance in Product Design}

According to the above, the design principles of behavioral interaction and emotional guidance in product design can be summarized into the following three points.

\subsection{Respect Existing Behaviors, Strengthen Behavioral Interactions, and Seek A Reasonable Interaction to Enhance Interaction Efficiency.}

People have strong behavioral interaction habits and abilities. People communicate through eye contact, facial expressions, body language, voice, biometrics and behavioral habits. As a design of humanized interaction, it is necessary to respect the attributes of human instinct behavior, and bring a new interactive experience in a well-known way to effectively arouse people's emotional resonance. Therefore, individual conscious or unconscious behavioral activities naturally become the trade-off point of the emotional interaction design innovation process. When the designer personally understands and seeks a possible and reasonable interaction behavior into the product design, it will definitely improve the interaction efficiency between users and products.

\subsection{Transform from One-way to Multi-directional Interaction Logic, Close to the User's Cognition and Achieve the Experience Goal}

The object-centered design is designed to achieve a reasonable distribution of the properties of the object itself. It has long been unable to meet the real needs of users. Therefore, the design principle need to transform from the object-oriented design pattern to the multi-directional design pattern oriented by "behavior" and "emotional psychology", from the simple "design of objects" to the "multi-directional interaction design of behavior and emotional psychology". At the same time, the logic of interaction also transforms from the one-way interaction logic of things and things, things and people to the multi-directional interaction logic of people and things in behavior, emotion and psychology. On the basis of understanding user behavior, emotional, and psychological logic, the product's usability and experience goals are achieved in a way that is closer to the user's cognitive style.

\subsection{Establish A Good Dialogue Relationship Between Users and Products, and Achieve A Virtuous Cycle of Interactive Experience}

Taking product and environment as the main role of user response, we construct a good interactive dialogue relationship between users and products in the three dynamic processes of using the product before, during and after the product. At the early stage, combined with environmental factors and clear product semantics, users can easily understand the physical and extended attributes of the product itself. Establishing a dialogue between users and products, and realizing the possibility of communication is the primary guarantee for forming a benign interactive experience relationship. In the mid-term stage, through the good setting and positioning of product functions, the in-depth interpretation of the use environment to form a benign communication situation between users and products to meet the needs of users to solve practical problems. Continuous and good interaction between the product and the user is realized by physical components such as sensors and indicator lights. Allow users to easily reach their goals at a lower learning cost and feel happy. Establishing user-led emotional connections is the top priority in building a benign interactive experience relationship. At the later stage, constantly blur the boundary between products and people (use and be used, the relationship between people and things), to achieve the integration of products and people (partnership), and ultimately promote a 
virtuous circle of interactive experience.

A good interaction relationship starts with user behavior research and combines the emotional elements behind different behaviors to create a unique use situation. Integrate element and method information into user behaviors, continuously improve the reliability and integration of products, and enable users to transition from the physical dimension of purely practical needs to the understanding of the meaning of creation and the spiritual dimension of interactive emotional experience [8]. As shown in Figure 5, in the scene of "emotion guided behavior and behavior enhance emotion as the core", people and things rely on "behavior" interaction to construct an interactive relationship and indirectly form a corresponding interactive experience. In addition, the interactive experience can also be directly generated by the emotional interaction between people and things, which in turn affects the construction of interactive relationships.

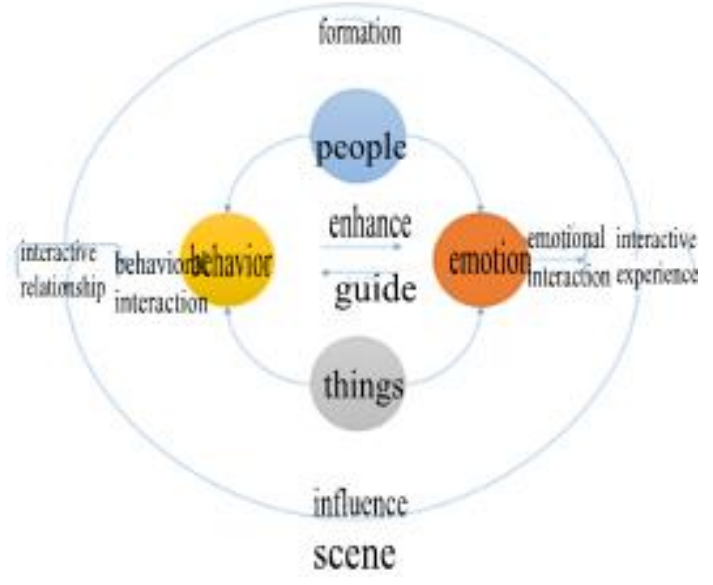

Figure 5 relationship map of benign interaction experience

\section{Conclusion}

All interaction design between users and products are not just about creating visual or physical effects, but also the overall experience [9]. The essence of interaction design is to seek a good emotional experience. The experience is both the starting point and the end of design. Experience is an effective mechanism for individual learning based on emotional systems to adapt to the environment. Sensory perception triggers users' expectation of the experience, evokes imagination and expectation of further behavior. Individual motivation triggers behavioral interaction, and constructs a benign experience in the multi-directional interaction of characters. Interactive behavior requires positive emotional drive. The designer adapts the product to the user's natural behavior under the premise of respecting human instinct and intuitive system, which is a more feasible strategy. Accurately grasping the behavioral interaction and emotional guidance theory in product interaction design plays an indispensable role in improving product user experience and enhancing interactive product capabilities and value.

\section{References}

[1] Liu Baisong, Xin Xiangyang and Liu Yuan. The Subconscious Interaction Design Method under Nerve Psychology [J]. Package Engineering, 2016 (10): 70-74.

[2] Xin Xiangyang. Interaction Design: From Physical Logic to Behavioral Logic [J]. Decoration, 2015 (1): 58 -62.

[3] Hua Sheng. Behavioral Psychology [M]. Beijing: Modern Press, 2016.

[4] SBakker and K Niemantsverdriet. The Interaction-Attention Continuum: Considering Various Levelsof Human Attention in Interaction Design [J]. International Journal of Design, 2016, 10.

[5] Carlson. Behavioral Physiology [M]. Shanghai: Shanghai People's Press, 2014. 
[6] MJM Kamil and SZ Abidin.Unconscious. Human Behavior at Visceral Level of Emotional Design [J]. Procedia Social and Behavioral Sciences, 2013, 105 (1): 149-161.

[7] Jesses James Garrett. Elements of User Experience [M]. Beijing: Mechanical Industry Press, 2007.

[8] Bruce Brown, Richard Buchanan, Carl Divosan and others. Design Issues: Experience and Interaction [M]. Beijing, Tsinghua University Press, 2017.

[9] Robert Brenner. The Critical Design [M]. Beijing: Renmin University of China Press, 2012. 